\title{
Multiferroic Magnetoelectric Composites and Their Applications
}

\author{
Mirza Bichurin, ${ }^{1}$ Vladimir Petrov, ${ }^{1}$ Shashank Priya, ${ }^{2}$ and Amar Bhalla ${ }^{3}$ \\ ${ }^{1}$ Novgorod State University, Veliky Novgorod 173003, Russia \\ ${ }^{2}$ University of Texas at San Antonio, San Antonio, TX 78249, USA \\ ${ }^{3}$ Virginia Polytechnic Institute and State University, Blacksburg, VA 24061, USA
}

Correspondence should be addressed to Mirza Bichurin, mirza.bichurin@novsu.ru

Received 2 September 2012; Accepted 2 September 2012

Copyright (C) 2012 Mirza Bichurin et al. This is an open access article distributed under the Creative Commons Attribution License, which permits unrestricted use, distribution, and reproduction in any medium, provided the original work is properly cited.

The progress in electronic technology is directly coupled with the advances made in materials science. Within the broad class of materials available today, functional materials provide unique opportunity for developing novel components and devices as their physical and chemical properties are sensitive to changes occurring in the environment such as temperature, pressure, electric field, magnetic field, and so forth. To exemplify, ferromagnetic and ferroelectric materials are presently utilized in a wide range of systems. A combination of ferromagnetic and ferroelectric materials provides a new class of functional materials, termed as magnetoelectrics. The magnetoelectric effect can be obtained through various other combinations and even in singlephase materials. The focus of this special issue is on fundamental understanding of the magnetoelectric (ME) multiferroic composites, their synthesis, and their application in some novel device architectures.

The ME effect can be defined as inducing the electric polarization in a material under an applied magnetic field, or vice versa, inducing the magnetization through an applied electric field. The effect was first observed in antiferromagnetic $\mathrm{Cr}_{2} \mathrm{O}_{3}$ at room temperature with $\mathrm{ME}$ voltage coefficient on the order of $20 \mathrm{mV} / \mathrm{cmOe}$. A composite material can be made magnetoelectric by combining magnetostrictive effect with the piezoelectric effect. In these composites, ME coefficient is dependent upon the elastic coupling occurring at the interface between piezoelectric and magnetostrictive phases. These magnetostrictive-piezoelectric composites provide significantly enhanced $\mathrm{ME}$ effect as compared to $\mathrm{Cr}_{2} \mathrm{O}_{3}$. Functional composites are defined by connectivity of materials such as 2-2 composite which is 2 dimensionally laminated, 3-0 composite which has dispersed particles in 3-dimensional matrix, and 3-1 composite which has 1-dimensional rods embedded in 3-dimensional matrix structures. Magnetostrictive-piezoelectric laminate composites with 2-2 connectivity have shown much higher $\mathrm{ME}$ coefficients than that of single-phase materials or particulate composites. In this issue, we provide detailed mathematical modeling approaches that can be used to describe the dynamic behavior of ME coupling in magnetostrictivepiezoelectric composites in wide frequency region, demonstrate practical transducer structures based upon these models, and discuss new applications.

Miniaturization of microwave devices is important for communication systems. Small-size and high-performance devices are necessary to reduce the cost and improve the functionality of system. There is a need for frequency tunable devices such as resonators, phase shifters, delay lines, and filters in the microwave and millimeter wave frequency regimes. Ferrites are used in tunable microwave and millimeter-wave devices, and the tunability is traditionally realized through the variation of a bias-magnetic field. This magnetic tuning could be achieved over a very wide frequency range, but is relatively slow and noisy, and requires high power for operation. Similar devices but with some unique advantages could be realized by replacing the ferrite with a ferrite-ferroelectric composite. The structures and models provided in this issue can be used to develop components that not only reduce the cost but also improve performance and application regimes.

The analytical expressions for the ME coefficient of magnetostrictive-piezoelectric laminates have been mostly derived under the assumption of homogeneity of electric, magnetic, and elastic field and by employing boundary 
conditions for mechanical stress in the integral meaning at the structure facets. Those expressions for ME coefficient are asymptotic at the lateral-to-transverse dimensions ratios tending to infinity which results from neglecting of the true boundary conditions for above-mentioned physical fields. However, the approach described in this issue will allow calculation of ME coefficients more accurately. The new model takes into account the fact that both the magnetostrictive and piezoelectric layers of the laminate are in a stressed state as a result of the bonding. The stressed state induces changes in many physical properties, especially, an appreciable change may occur in the magnetic and magnetostrictive characteristics of the magnetostrictive layer made of a ferromagnetic nickel with cubic symmetry. The numerical simulations provided in this issue will lead towards a robust, physically reasonable, and accurate modeling technique. These new approaches are capable of addressing a wide range of the ME laminate designs with different geometries, materials, and loading configurations.

The methodology for improving the magnetoelectric coefficient of the sintered composite is discussed in this issue along with detailed composition-microstructure-property relationships over broad range of systems. The role of new material constant $n$ is discussed that can be used to tailor the magnitude of piezoelectric voltage constant. According to the proposed model, as the magnitude of $n$ decreases towards unity a giant enhancement in the magnitude of the $g$ constant can be obtained. The experimental data shown in the paper was found to follow this prediction. Effect of matrix grain size on the magnetoelectric coefficient particulate composites is described. There is a critical grain size below which the piezoelectric charge constant, dielectric constant, and piezoelectric voltage constant drop rapidly. Optimization studies with respect to magnetostrictive to piezoelectric thickness ratio, magnetic field orientation, multilayering, and texturing are reported and experimental results are provided that should help the materials designers to develop better composites. Further effect of nanostructures in the form of core-shell composites is described. We believe these studies capture many important aspects of the magnetoelectric composite design using sintering methods.

ME materials have been investigated to find applications in sensors, transducers, actuators, energy harvesters, and servomechanism. Experimental and analytical results on ME laminates have shown relatively large ME output voltage, but the peak in ME coefficient occurring at the optimum DC bias is generally sharp. This limits their ability to be utilized for AC magnetic field sensing when the optimized DC condition is shifted by external noises. Moreover, high ME coefficient generally occurs near the electromechanical resonance (EMR) frequency. This narrow bandwidth also poses problem for stable sensing in a limited frequency range. In order to overcome the problems of limited DC and AC magnetic field ranges associated with laminates, one of the papers in this issue described new transducer structures that could lead to flat ME responses as a function of DC magnetic field and AC magnetic field frequency. Various structural and geometrical parameters were investigated to understand the variation in ME coefficient with applied
DC magnetic bias. By controlling laminate dimensions, number of magnetostrictive layers and gradient geometry, the correlation between the overall deformation and peak position in ME coefficient was found. The broad/wideband ME behavior with near flat ME response was demonstrated by designing a dimensionally gradient bimorph structure and combining with laminate configuration. We expect these results will be useful for application engineers working towards development of current probe, magnetic field sensing, and ME energy harvester.

Numerous efforts are being made to fabricate the "advanced memory", which is simultaneously nonvolatile, dense, robust, fast, and less energy expensive. Ferroelectric or FE (ferromagnetic or FM) materials, owing to their switchable polarization (magnetization) states with remnant polarization (magnetization), have already demonstrated their importance in the data storage industry by allowing storage of binary information in their two polarization (magnetization) states. In the commercially available magnetic memory device (MRAM), data is written by switching the magnetic states $( \pm \mathrm{M})$ upon application of a magnetic field, while to read data, one exploits variation of magnetoresistance in the magnetic states. Being hard ferromagnets, the materials in the MRAMs possess high coercivity resulting in large magnetic field requirement for switching magnetic states and thus consume large amount of energy. In contrast, ferroelectric memories (FeRAMs) possess faster writing speeds via polarization switching and are energy efficient. However, there is limitation on their size and they exhibit slow reading speeds due to their destructive read operation and subsequent reset. Therefore, a memory device with the combination of best functionalities of FeRAMs and MRAMs (ferroelectric write and magnetic read operations) would effectively enhance the writing speed and reduce the energy consumption. Moreover, device miniaturization can further lead to reduced energy consumption and higher speeds. Unfortunately, any further improvement in the bit density in the MRAMs/FeRAMs is hampered in the FM/FE materials by presence of only two switchable states. From this perspective, multiferroic and magnetoelectric materials present new possibilities towards enhancing data densities by many folds and thus pave the opportunity towards the fabrication of the next generation memory devices. Multiferroics and magnetoelectrics can make use of both the functionalities of FeRAMs and MRAMs, independently, to store binary data. Further, coupling between electrical- $( \pm \mathrm{P})$ and magnetic- $( \pm \mathrm{M})$ order parameters in these materials provides a possibility of possessing additional functionalities such as electrical (magnetic) control of magnetization (polarization), enabling design of futuristic multistate memory devices with electrical writing and nondestructive magnetic reading operations. In this issue, a detailed review is presented covering multiferroic and magnetoelectric materials that can be used to design high-density and efficient nonvolatile memory elements for memory devices. Material selection process with respect to device fabrication approaches is also discussed to address the compatibility issues. We believe this topic will become continually important and there is need for solving the basic problems restricting the realization of better memories. 
All these topics combined provide an excellent coverage of most important facets of magnetoelectric multiferroic materials that will guide the readers towards better understanding of this phenomenon and lead towards design and development of better components and devices. Ultimately, our goal for this issue is to act as a facilitator towards the transition of the magnetoelectric multiferroic materials into practical devices.

Mirza Bichurin Vladimir Petrov Shashank Priya Amar Bhalla 

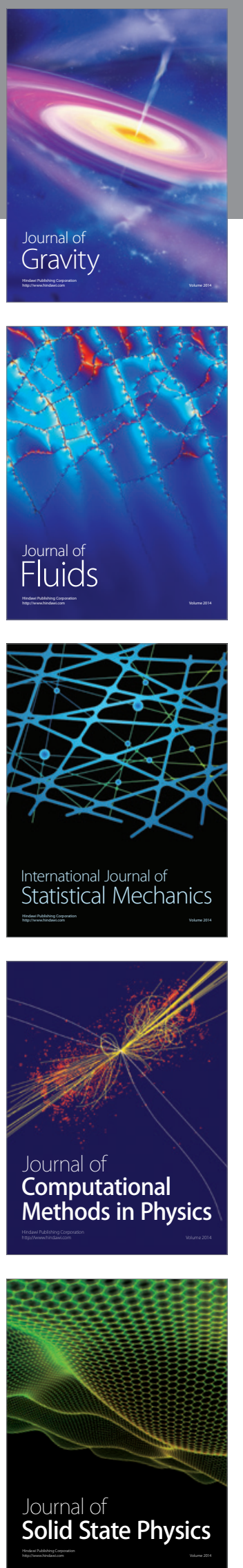

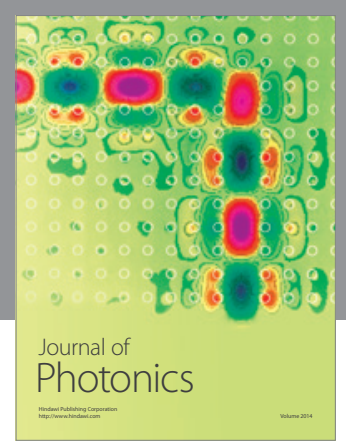

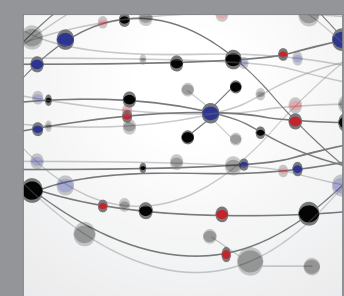

The Scientific World Journal
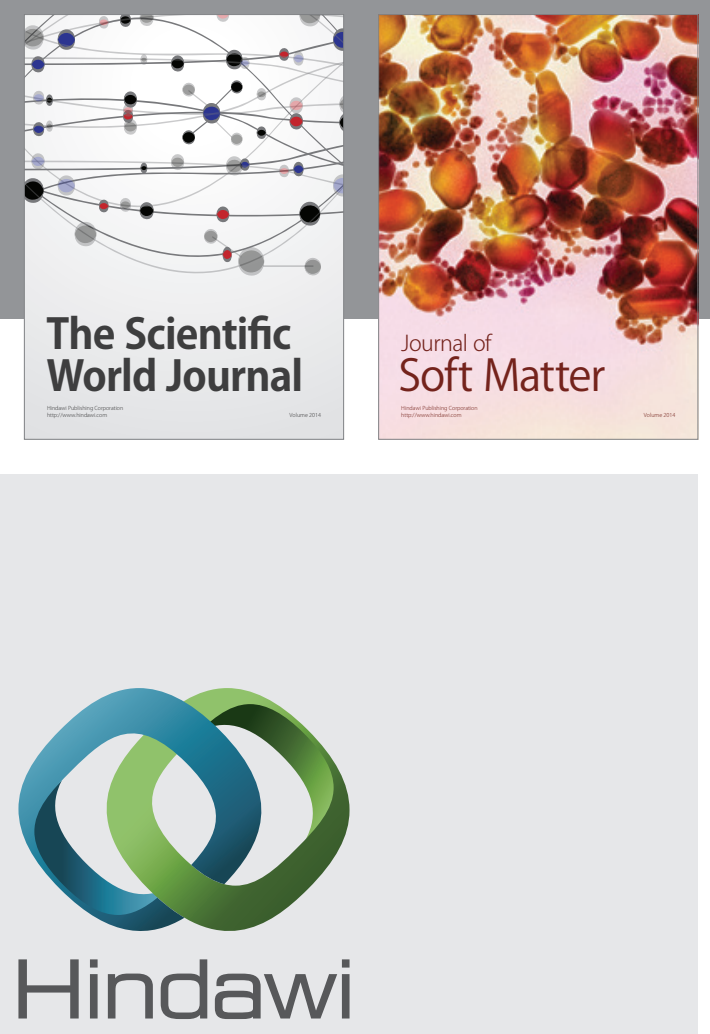

Submit your manuscripts at

http://www.hindawi.com
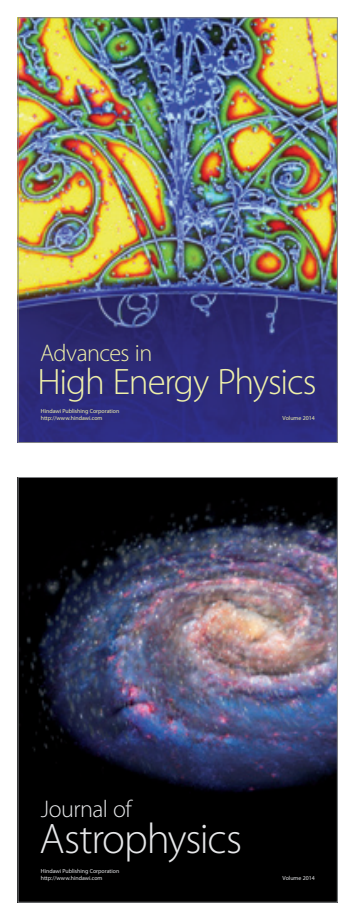
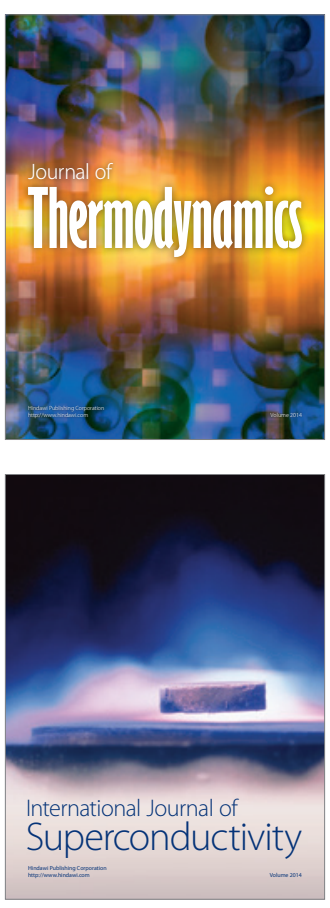
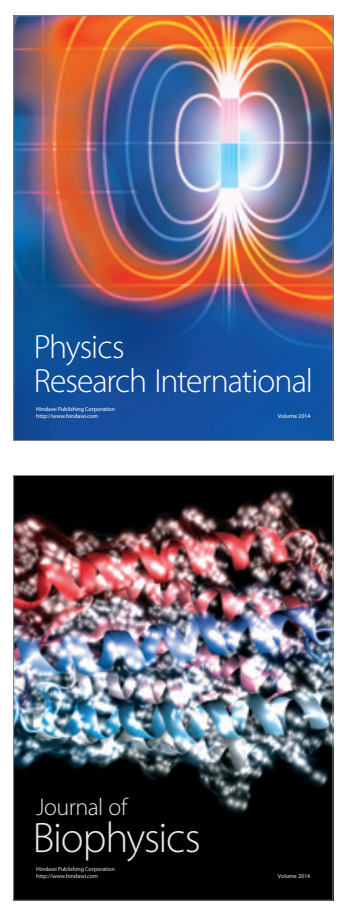
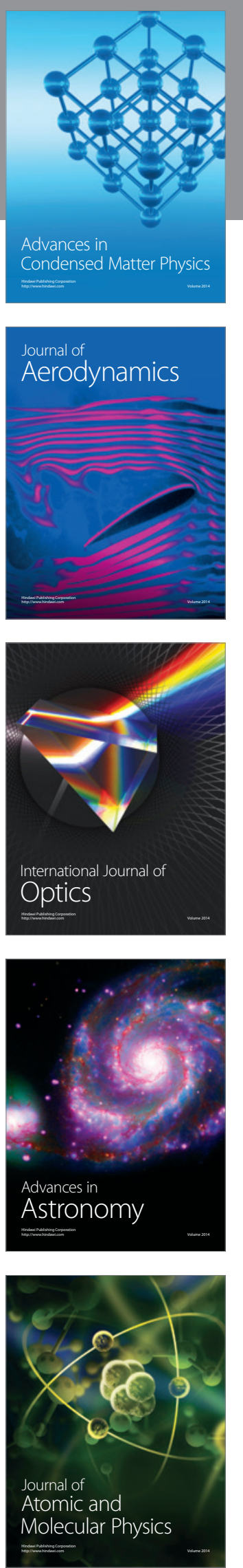